\title{
CFD BASED EVALUATION OF THE LOCK-IN PHENOMENON OF A BRIDGE UNDER WIND LOAD
}

\author{
AdelinoV. Lopes ${ }^{1}$, Álvaro Cunha ${ }^{2}$ and Luís M. C. Simões ${ }^{1}$ \\ ${ }^{1}$ Department of Civil Engineering \\ Faculty of Sciences and Technology of the University of Coimbra, Coimbra, Portugal \\ \{avlopes, lcsimoes\}@dec.uc.pt \\ ${ }^{2}$ Department of Civil Engineering \\ Faculty of Engineering of the University of Porto, Porto, Portugal \\ acunha@fe.up.pt
}

Keywords: Bridge Aeroelastic Analysis; Serviceability Limit States of Vibrations; Vortexshedding; Lock-in; CFD; FVM; Geometric Non-Linear Analysis; FEM.

\begin{abstract}
This paper presents a methodology for the numerical analysis of the serviceability conditions of slender structures under wind actions, having in consideration the possibility of occurrence of synchronized phenomena (lock-in) due to vortex-shedding. The computational model used in the aeroelastic analysis of slender structures is based on the appropriate conjugation of an algorithm of Computational Fluid Dynamics (Control Volume Method) with an algorithm of linear or geometrically non-linear analysis of structures. This new methodology was developed in order to evaluate the ultimate limit states of instability, specifically the critical velocity of aeroelastic instability. Now, this methodology is also applied to the safety checking of the vibrations' serviceability limit state of a simply supported bridge with a rectangular cross-section $(B / D=6)$, under wind load considering their fundamental frequency. In particular, it will be evaluated the range of this synchronized phenomena, the peak value of acceleration obtained and: i) the time to start the phenomena; ii) the fully developed time; iii) and the corresponding time step. Some of the most interesting results associated with the evaluation of the corresponding response acceleration are also presented and compared with available human body acceptance criteria for vibrations (comfort evaluation) present in the technical literature.
\end{abstract}




\section{INTRODUCTION}

The analysis of the dynamic behaviour of long span cable-stayed and suspension bridges under wind excitation is usually performed on the basis of experimental tests on physical models in wind tunnels [1], which allow foreseeing the possibility of loss of stability in the real structure.

As an alternative to such procedure, some numerical methodologies have been developed, specifically adapted to each aeroelastic phenomenon, namely in terms of flutter analysis $[2,3]$, though they are still based on some coefficients (flutter derivatives or Scanlan coefficients) whose evaluation still involves usually the use of experimental tests (forced or free vibration tests).

An attempt to overcome such limitations consists in using algorithms of Computational Fluid Dynamics, that permit the numerical simulation of the air flow around the deck crosssections. This type of applications has been considered for the evaluation of force or Scanlan coefficients, but not for integral aeroelastic analyses of slender bridge decks, taking into account the temporal evolution of the dynamic flow-structure interaction.

After recent progress in computer technology, the authors could develop and implement a new numerical methodology for the aeroelastic analysis of slender structures $[4,5,6]$. This computational tool is a time incremental approach based on two coupled numerical algorithms: one of them determines the fluid flow action and the other one evaluates the structural response. The Finite Element Method (FEM) is used to model the structural dynamic behaviour, which can be idealised as geometrically non-linear. The numerical procedure used to calculate the fluid flow and its action on structures is based on the Finite Volume Method (FVM). It is considered a viscous incompressible unsteady turbulent bidimensional air flow solved on a structured control volume mesh. The mentioned algorithm uses an iterative sub-process to achieve the correspondence between aeroelastic forces and structural movements at every time step.

However, most of the applications performed deal with the evaluation of the critical velocity, also known as critical flutter velocity, which must be high enough. This procedure can be understood as a verification of the structural safety in terms of ultimate limit state. But, as mentioned by the Eurocode [7], it may be also needed to verify the serviceability limit states of vibrations caused by wind action. In the particular case of very flexible bridges under wind loads, this verification can be done in terms of undesirable effects for users (discomfort), comparing the evaluated acceleration (or velocity) root mean square (or peak) values with human body acceptance criteria for vibrations. As it is known, these vibrations caused by wind action can be inconvenient for users when there is synchronization between the frequency of vortex shedding and some fundamental structural frequency. In order to prevent this resonance phenomenon, Eurocode suggests moving away, as much as necessary, from structural frequencies, which can present some difficulties. On the other hand, it is worth mentioning that significant oscillations due to vortex-shedding have been observed in several long span bridges recently constructed [8].

In this context, this paper presents the application of the above mentioned computer algorithm to the evaluation of the serviceability conditions of a simply supported bridge with a rectangular cross-section $(\mathrm{B} / \mathrm{D}=6)$, under wind load considering their fundamental frequency. In particular, it will be evaluated the range of this synchronized phenomena, the peak value of acceleration obtained and: i) the time to start the phenomena; ii) the fully developed time; iii) and the corresponding time step. The unknown peak values are achieved by checking the maximum amplitude in the range of synchronized phenomena (lock-in) due to vortexshedding. Some of the most interesting results associated with the evaluation of the corre- 
sponding acceleration peak values of movements are also presented, and compared with available human body acceptance criteria for vibrations (comfort evaluation) listed in Bachmann [9], ISO 2631 [10], CEB [11], DIN 4150 [12] and NRCC [13].

\section{NONLINEAR COUPLED FLUID-STRUCTURE AEROELASTIC ANALYSIS}

The computational algorithm developed to simulate aeroelastic phenomena in slender structures is a time incremental approach based on two numerical algorithms working together: one of them determines the fluid flow action and the other one evaluates the structural response. The numerical procedure used to calculate the fluid flow and its action on structures is based on the FVM. The FEM is used to model the structural dynamic behaviour, which can be idealised as geometrically non-linear.

\subsection{Fluid flow simulation}

The implemented program, based on the Finite Volume Method [14, 15, 16], is suitable to simulate incompressible and isothermal bidimensional unsteady fluid flows around obstacles. It is assumed that the flow's domain may be discretised in a Cartesian and structured control volume mesh, whose faces have vertical and horizontal directions.

The equations taken from the integration of general transport equations in differential forms are discretised by using the hybrid differentiation scheme. In order to reduce false diffusion, a refined mesh around boards of the obstacle is considered and the QUICK (Quadratic Upstream Interpolation for Convective Kinematics) differentiation scheme is also used in deferred correction context [16]. The stability is preserved by the use of base scheme (hybrid) to set up all coefficients of every equation, and by taking into consideration all the differences to the adequate scheme in source term. Due to their complexity and extensity, hybrid and QUICK coefficients, as source and deferred correction related equations, are not indicated here, but can be found in Lopes [4, 6].

Alternate value field resulting from first derivatives of pressure or velocity is avoided on the basis of a staggered grid approach, which is the basis of the SIMPLE (Semi-Implicit Method for Pressure-Linked Equations) procedure also used to ensure correct linkage between pressure and velocity field values. All these methods are iterative algorithms and, when other scalars (like turbulent quantities) are coupled to the momentum equations, the calculation has to be done sequentially. In order to ensure stability of the iteration process of this strongly non-linear problem, all these methods require under-relaxation what will be mentioned.

In this algorithm, the high Reynolds number $k-\varepsilon$ turbulence diffusion model is applied to simulate the flow turbulence $[17,18,19]$.

It should be noted that the probability of instability grows as the flow velocity increases (high convective). In this case, it is possible to use the same under-relaxation factors in order to get both stability and a solution procedure for transient calculations. Considering this procedure, the corresponding time interval for convective flows can be set up from the following inequality

$$
\Delta t \leq \frac{\min \left(\delta_{n}\right)}{2 U}
$$

where $\delta_{n}$ represents the distance between the central points of two adjacent control volumes and $U$ represents the velocity of the free flow.

The model to simulate fluid flows is completed by defining boundary conditions, which can be separated into two parts: one of them for obstacle walls and the other for all limits of 
the considered external flow's domain (inlet and outlet). In the first case, the followed methodology depends on the sub-region where every particular node neighbourhood at each wall is situated [20]. Considering the turbulent boundary layer which can be separated in two others (viscous and logarithmic), the high Reynolds number $k-\varepsilon$ turbulence diffusion model can be used by defining particular values for these nodes, if the first node from the wall is located in logarithmic sublayer. When the first node is located in the viscous sublayer it is used a low Reynolds number version. All relevant equations and values used for modulation of turbulence and for defining boundary conditions at both obstacle walls and remaining boundary conditions in inlet and outlet regions can also be consulted in Lopes [4, 6].

The convergence criterions for pressure-correction equations and for the remaining equations are respectively

$$
\begin{aligned}
& \frac{1}{n} \sum_{n} \frac{\left\|{ }^{i} b\right\|}{\rho U} \leq 10^{-4} \\
& \frac{1}{n} \sum_{n} \frac{\left\|{ }^{i} \phi-{ }^{i-1} \phi\right\|}{\phi_{\text {inlet }}} \leq 10^{-4}
\end{aligned}
$$

where $n$ is the number of control volumes, $\rho$ is the density of the fluid, ${ }^{\mathrm{i}} b$ is the source term at the $i^{\text {th }}$ iteration, ${ }^{i} \phi$ is the field of the generic property value calculated at the $i^{\text {th }}$ iteration and $\phi_{\text {inlet }}$ is the correspondent field value in the inlet domain.

\subsection{Structural analysis}

The Finite Element Method is used to model the structural behaviour [21, 22, 23]. The simulation of the dynamic behaviour is based on the incremental Newmark Method and the corresponding integration parameters are set up according to Newmark's initial proposal (constant-average-acceleration-method). Structural damping is introduced by assuming a Rayleigh damping matrix, where the mass and stiffness matrix coefficients are evaluated by adopting two particular modal damping factors. The numerical procedures, based on an Updated Lagrangian formulation, allow the consideration of global large displacements (geometrical non-linear behaviour). However, small element deformations were assumed to evaluate the structural response.

In this incremental algorithm, the main purpose at every incremental time step $\Delta t$ consists in reducing the non-balanced structural forces as much as possible, with the intention of obtaining the updated structural shape ${ }^{t+\Delta t} a$. With this intend, this process involves an iterative sub-process with the purpose of evaluating the global increment of displacements ${ }^{t+\Delta t} \Delta a$ at the corresponding $\Delta t$, which will be added to the displacements at the previous time instant ${ }^{t} a$.

In any incremental time interval, the convergence criterion for non-balanced forces at the $i^{\text {th }}$ iteration is

$$
\frac{1}{n} \sum_{n} \frac{\left\|{ }^{i} a-{ }^{i-1} a\right\|}{L_{r e f}} \leq 10^{-8}
$$

where $n$ is the number of degrees of freedom and $\mathrm{L}_{r e f}$ is a reference length (for instance, maximum structural dimension). 


\subsection{Aeroelastic algorithm}

A structural system is submitted to several forces when immersed in a fluid flow. If the structure is flexible, these forces are called aeroelastic forces, and they depend, not only on the flow characteristics around the structural system, but also on the structural response [24].

The present algorithm uses an iterative sub-process to achieve the convergence between aeroelastic forces and structural movements at the end of every time step.

The iterative sub-process begins based on the prediction about the movements at the end of each time step, by using the following linear extrapolation

$$
{ }^{t+\Delta t} \ddot{a}_{k}=2^{t} \ddot{a}_{k}-{ }^{t-\Delta t} \ddot{a}_{k}
$$

Then, the algorithm solves the flow equations and calculates the aeroelastic forces, which allows the determination of the corresponding structural movements. If those movements are not in good agreement with the predictions, these predictions must be corrected and this subprocess should be reinitiated until convergence is achieved. The convergence criterion is similar to equation (3).

Due to the characteristics of bidimensional fluid flow simulation, this algorithm considers several transversal cross sections along the slender part of the structure where the aeroelastic forces are calculated. This simplified procedure assumes that the flow is normal to the longitudinal axis of the slender structure. Moreover, the flow around one section is simulated by itself and is considered independent from the other sections.

As it is mentioned above, this aeroelastic algorithm does not consider the threedimensional flow effects, which constitutes the weakest feature of the presented fluidstructure model. However, it is expected that the three-dimensional effects, associated to the variation of the flow and structural geometry along a third spatial dimension, are not very significant for long cable-stayed or suspension bridges. This means that there are not considerable effects coming from the flow parallel to longitudinal deck axis, and the geometry variations are only localised in a few sections, which is probably insufficient to deeply change the characteristics of the global dynamic aeroelastic forces acting on the bridge deck.

The structural movements in fluid flow are modelled indirectly by changing the velocity components $\left(v_{1}\right.$ and $\left.v_{2}\right)$ of the fluid flow at external inlet boundary domain as described in Lopes $[4,6]$.

\section{ANALYSIS OF THE SERVICEABILITY CONDITIONS}

The effects (movements) originated on flexible structures by the aeroelastic action of wind have oscillatory features, evidencing in general an amplitude and a predominant frequency.

Regarding ISO 4866 [25] related to vibration of buildings, the typical range of structural response due to wind action will be from $0.1 \mathrm{~Hz}$ to $10 \mathrm{~Hz}$, and the measuring quantities should be in acceleration. In this case, vertical components are often dispensed and only rotational and translational ones should be always considered for the evaluation of effects of vibration on buildings. Otherwise, in bridge domain, the vertical component of vibration is also very important in order to evaluate the effects on people.

Considering the wind action on structures, the general vibration condition could have two main sources: one due to external instability of the free fluid flow (velocity oscillations), and other caused by the internal instability (obstacle geometry) and obstacle movements.

In bridges field, the own vibration of the free fluid flow velocity can itself develop a phenomena, known as buffeting, which usually will be not so important for small bridges as for users themselves. In this particular case of vibrations in the wind direction, the structural 
damping is significantly incremented by the aeroelastic damping [26] arising from internal flow conditions, which does not allow large amplitude of oscillations.

Considering now the mean flow, if the free velocity is bigger than a certain value, called critical velocity, the structural system has divergent oscillations, commonly known as flutter phenomenon. After enough time in these conditions, the structure may have developed enlarged straining, plasticity included, or even may have fallen down. From the designer point of view, it one searches to have the highest possible flutter velocity.

On the other hand, when the free fluid flow velocity is less than the critical velocity, the bridge system also shows some continuous vibrations, whose characterisation depends on vortex-shedding of the cross section. Generally, this vibration does not develop enlarge straining, but can lead to unacceptable vibration exposure, from human comfort point of view, or even materials fatigue.

These vibrations become important (large amplitudes) when the aeroelastic forces are synchronised with a structural frequency, inducing resonance effects. It is worth mentioning that, the aeroelastic forces are associated to the vortex-shedding through the Strouhal number St. This vortex-shedding phenomena depends not only on the obstacle geometry, but also on the free fluid flow velocity and mainly, on the obstacle movements. As it is shown later, this synchronisation will appear for a relatively wide range of free fluid flow velocity. This synchronised phenomenon, called lock-in, is well known in aeroelastic field. In principle, the corresponding free airflow velocity of reference will be $\mathrm{U}=\mathrm{fs} . \mathrm{D} / \mathrm{St}$, where fs is the structural frequency and D represents the dimension in normal-wind direction. Eurocode 1 [7] suggests avoiding this synchronisation, but this phenomenon has been observed [8] as the free wind velocity can vary between wide limits, and specially because there is a widely range of wind velocities where this phenomena can be developed, as pointed out before. Modifying the geometry of the cross section in order to change the vortex shedding behaviour of the disturbing bridge can modify the amplitude and frequency of oscillations but does not prevent the phenomenon.

So, considering the possibility of occurrence of these effects, it seems interesting to develop a methodology for the analysis of the serviceability conditions. In this case, the mentioned analysis can start by determining the range where the synchronised phenomena will happen. After that, it will be necessary to evaluate the induced levels of oscillations (acceleration or velocity values of movements) in this range in order to know the maximum amplitude of oscillation which can be compared with available human body acceptance criteria for vibrations (comfort evaluation) listed in Bachmann [9], ISO 2631 [10], CEB [11], DIN 4150 [12] and NRCC [13]. Moreover, it will be interesting to know the related characteristics as: i) the step time needed to start the mentioned synchronisation; ii) the step time to get the levels of maximum oscillations; iii) and the phenomena after starting.

The acceleration peak values of structural movements are evaluated by using the described program. With regard to the establishment of human body acceptance criteria for vibrations, two points must be referred: (i) there are not limit values set up specifically for bridge cases under wind action; (ii) some of acceptance criteria are developed taking into account sound wave tests and/or they are applicable to higher frequency phenomena; (iii) in general, human body acceptance criteria for vibrations depend on body position (standing, sitting, or lying), on activity (working, walking, resting, etc), on main oscillations' direction (vertical or horizontal), on magnitude and frequency of vibrations, and also on the duration of the exposure. The last parameter is certainly one the most important. In the present case it is assumed that the users of the bridge are standing, resting, and the vibrations are vertical.

Two widely used codes are ISO 2631 [10] and DIN 4150 [12]. The International Standard ISO 2631 covers all effects on people from random and shock vibrations as well as harmonic 
vibrations in both vertical and horizontal directions and it deals with the exposure times. The criteria are formulated in terms of a measured effective acceleration, i.e. the root mean square value $R M S$ of accelerations. Note that the RMS is 0.707 times the corresponding peak value for pure sinusoidal vibrations. Figure 1 shows the fatigue-decreased proficiency boundary on $\mathrm{z}$-acceleration which applies to the level at which recurrent vibrations cause fatigue to working personnel with consequent reduction in efficiency. The exposure limit, which defines the maximum tolerable vibration with respect to health and safety, is obtained by multiplying by two the fatigue-decreased proficiency boundary.

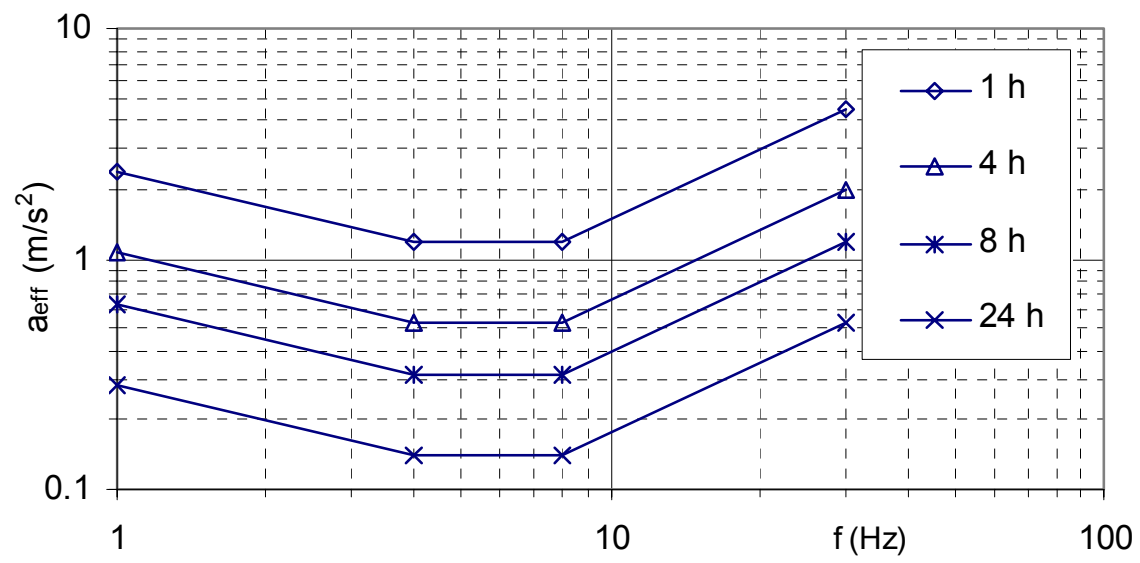

Figure 1: Bounds on z-effective acceleration for fatigue-decreased proficiency.

The German Standard DIN 4150 deals with the effects of vibrations from mostly external sources on people in residential buildings. The measured acceleration value of vibration $a$ together with the frequency $f$ is used to calculate an empirically derived intensity of perception factor $K B$ using the formula

$$
K B=\frac{20.3 a}{\sqrt{1+0.032 f^{2}}}
$$

The acceptable $K B$ value is upper limited to about 0.3 for continuous horizontal vibrations on residential buildings for frequencies greater than $1 \mathrm{~Hz}$.

Table 1 resumes other information of some human body acceptance criteria for the found vibrations, where $a$ means the peak value of structural acceleration and $g$ is the gravity acceleration. 


\begin{tabular}{|c|c|c|c|}
\hline Reference & Human body ac & criteria & Obs. \\
\hline \multirow{2}{*}{ Bachmann [9] } & Perceptible & $a \leq 5 \% g$ & \multirow{2}{*}{ Pedestrian structures } \\
\hline & Uncomfortable & $5 \% \mathrm{~g}<\mathrm{a} \leq 10 \% \mathrm{~g}$ & \\
\hline \multirow{3}{*}{$\begin{array}{l}\text { CEB [11] } \\
\text { (p. 231) }\end{array}$} & Perceptible & $\mathrm{a}=0.1 \% \mathrm{~g}$ & \multirow{3}{*}{$\begin{array}{l}\text { Vertical harmonic vibra- } \\
\text { tion for standing persons }\end{array}$} \\
\hline & Disturbing & $a=5.5 \% g$ & \\
\hline & Intolerable & $a=18 \% g$ & \\
\hline \multirow{3}{*}{$\begin{array}{l}\text { CEB [11] } \\
\text { (p. 83) }\end{array}$} & Perceptible & $0.5 \% \mathrm{~g}<\mathrm{a} \leq 1.5 \% \mathrm{~g}$ & \multirow{3}{*}{ Building structures } \\
\hline & Annoying & $1.5 \% \mathrm{~g}<\mathrm{a} \leq 5 \% \mathrm{~g}$ & \\
\hline & Very annoying & $5 \% \mathrm{~g}<\mathrm{a} \leq 15 \% \mathrm{~g}$ & \\
\hline \multirow{2}{*}{ NBCC [13] } & Perceptible & $a \leq 4 \% g$ & \multirow{2}{*}{ Rhythmic activities } \\
\hline & Uncomfortable & $4 \% \mathrm{~g}<\mathrm{a} \leq 7 \% \mathrm{~g}$ & \\
\hline
\end{tabular}

Table 1: Human body acceptance criteria for vibrations.

\section{APPLICATION}

\subsection{Description of the problem and evaluated results}

This methodology was applied to the analysis of the serviceability conditions of a simply supported bridge, with a rectangular deck cross-section $\mathrm{B} / \mathrm{D}=6$, such as it is illustrated in figure 2. This structure was modelled considering 10 beam elements, of same length, whose mechanical properties are presented in table 2. Table 3 shows the first four natural frequencies and respective mode types. Structural damping was idealised on the basis of a Rayleigh damping matrix, whose composition was determined by assuming modal damping factors of $0.5 \%$ for the first vertical bending and torsional modes. The evaluation of the aeroelastic forces was made by simulating the fluid flow around three cross-sections: 3, 6 and 9 (see figure 2).

The fluid (air at standard conditions) flow mesh was built by using $99 \times 54$ control volumes, with a minimum and a maximum dimensions of $5.24 E-2 m$ and $64 E-2 m$ according to direction $O x_{1}$, and for direction $O x_{2}, 5.27 E-2 m$ and $77 E-2 m$, respectively. Such as it is indicated in figure 3, the distances from the faces of the deck's cross section to the boundary domain were fixed so as to obtain forces not dependent upon those distances. Globally, in this example, the aeroelastic algorithm running in a Pentium IV based PC takes about 10 minutes to simulate only one real second. 

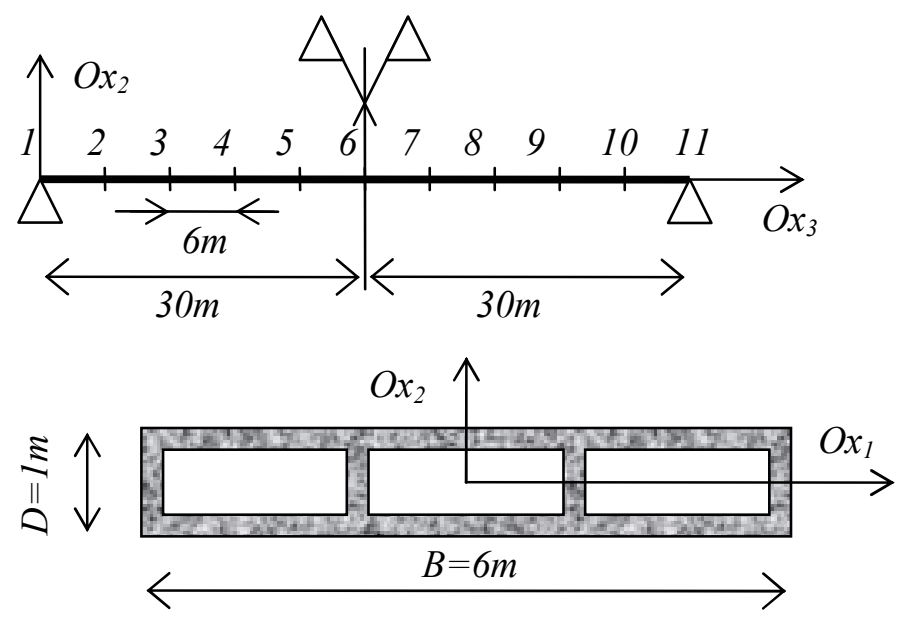

Figure 2: Geometry of the simply supported bridge.

\begin{tabular}{||c|c||}
\hline Axial stiffness $(\mathrm{EA})$ & $4.5 \mathrm{E} 7 \mathrm{kN}$ \\
\hline Ox $_{1}$ flexural stiffness $\left(\mathrm{EI}_{1}\right)$ & $7.8 \mathrm{E} 6 \mathrm{kN} . \mathrm{m} 2$ \\
\hline $\mathrm{Ox}_{2}$ flexural stiffness $\left(\mathrm{EI}_{2}\right)$ & $1.6 \mathrm{E} 8 \mathrm{kN} . \mathrm{m} 2$ \\
\hline Torsional stiffness $\left(\mathrm{GI}_{\mathrm{p}}\right)$ & $2.0 \mathrm{E} 6 \mathrm{kN} . \mathrm{m} 2$ \\
\hline \hline
\end{tabular}

Table 2: Mechanical characteristics of the simply supported bridge.

\begin{tabular}{|c|c|c|}
\hline Mode & Type & Freq. (Hz) \\
\hline \hline 1 & 1st vertical & 0.629 \\
\hline 2 & 2nd vertical & 2.52 \\
\hline 3 & 1 st horizontal & 2.84 \\
\hline 4 & 1st torsional & 3.17 \\
\hline
\end{tabular}

Table 3: Dynamic characteristics of the simply supported bridge. 


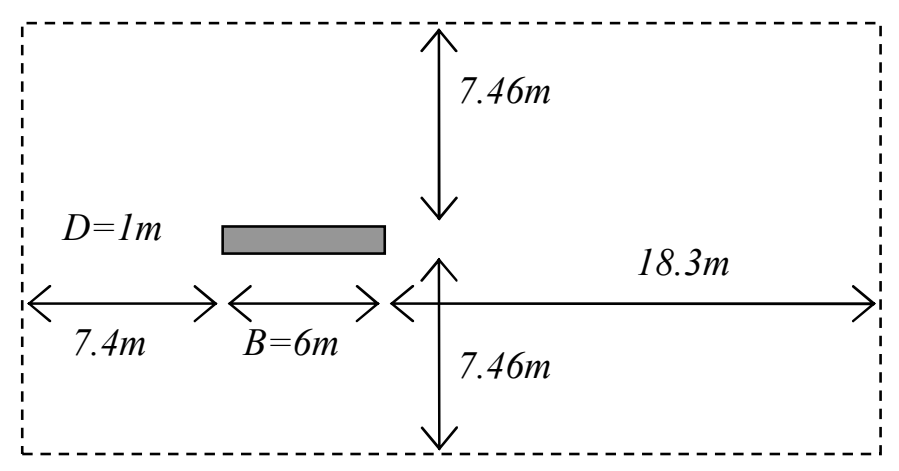

Figure 3: Domain of the fluid flow simulation.

Simulation 1: $\mathbf{R e}=\mathbf{4 E 5}$. Initially, with the purpose of analysing the lock-in phenomenon around the fundamental frequency, it is considered only the free fluid flow velocity corresponding to the first vertical (fundamental) structural frequency, defined by Strouhal velocity. So, for $f_{S}=0.629 \mathrm{~Hz}$ and taking into account approximately $S t=0.105$, this free flow velocity will be approximately $U=6.02 \mathrm{~m} / \mathrm{s}(R e=4 E 5)$, where the dimensionless Reynolds number $R e=\rho U D / \mu, \rho$ and $\mu$ represent the density and the dynamic viscosity of the fluid. For dynamic structural analysis, the used incremental time step was $1 E-2 s$. Moreover, for fluid flow simulations and for each of these used intervals, five incremental time steps were adopted.

The simulation considers two phases: the structure is fixed in a previous instance and is free to deform in a second one. Before releasing the structure, the velocity of the free flow is elevated to the pre-defined value and the simulation is led to a stable condition according to oscillatory aeroelastic forces. After that, the time account starts and the structure is liberated.

It is presented in table 4 some static values corresponding to the mean value, the corresponding amplitude and the predominant frequencies of the aerodynamic force coefficients, when the flow around a fixed cross-section is considered. CFi represents the coefficient of the aerodynamic force according to direction $i, \Delta$ the amplitude of oscillation and $S t$ is the adimensional frequency predominant, i.e. the Strouhal number. It is worth to write down that, after reaching these cited flow velocity, the aerodynamic forces show to have small amplitudes of oscillation, which tend to become almost zero during the simulation. But, after a while, they come back to assume relevant values. In table 4, these last gotten values are indicated after stabilization of the corresponding amplitude.

In table 5 and figures 4-11 are presented some of the most significant results concerning displacements and aeroelastic forces at the mid-span section, i.e. section number 6 in figure 2 , after releasing the structure.

It should be referred that, initially, after being released, the structure suffers some disturbance, mainly in the horizontal direction, due to drag. In this simulation, this process is prolonged for about 30s. After this phase, the structure shows a consistent increasing of the vertical oscillation amplitude until reaching an unalterable value of about 150 s time. The vertical aeroelastic forces develop a similar process, slightly advanced in about 5s. However, these oscillations evidence a constant frequency of $0.62 \mathrm{~Hz}$. The remaining movements and forces oscillate with small amplitude, but they are conditioned by the Strouhal frequency. It should be pointed out that the value of the taken maximum vertical acceleration amplitude is $0.30 \mathrm{~m} / \mathrm{s} 2$. The corresponding derived intensity of perception factor KB value is 6.1 . 


\begin{tabular}{|c|c|c|c|c|c|c|c||}
\hline$R e$ & $U(m / s)$ & $\Delta t(s)$ & $C F 1$ & $\Delta C F 1$ & $\Delta C F 2$ & $\Delta C F 12$ & $S t$ \\
\hline $4 \mathrm{E} 5$ & 6.02 & $2 \mathrm{E}-3$ & 1.125 & 0.0087 & 0.072 & 0.058 & 0.107 \\
\hline
\end{tabular}

Table 4: $\mathrm{Re}=4 \mathrm{E} 5$. Relevant values got when the cross-section is at rest.

\begin{tabular}{|c|c|c|c|c|}
\hline Dependent variable & Time interval & Amplitude & $\begin{array}{l}\text { Frequency } \\
(\mathrm{Hz})\end{array}$ & $\begin{array}{l}\text { Max. spec- } \\
\text { trum value }\end{array}$ \\
\hline \multirow{2}{*}{ Deflection } & $00-100 \mathrm{~s}$ & Growing & 0.621 & $9.8 \mathrm{e}-4$ \\
\hline & $500-600 \mathrm{~s}$ & $\pm 2.0 \mathrm{e}-2 \mathrm{~m}$ & 0.621 & $1.7 \mathrm{e}-2$ \\
\hline \multirow{2}{*}{ Vertical Acceleration } & $00-100 \mathrm{~s}$ & Growing & 0.621 & $2.0 \mathrm{e}-2$ \\
\hline & $500-600 \mathrm{~s}$ & $\pm 0.300 \mathrm{~m} / \mathrm{s}^{2}$ & 0.621 & $3.9 \mathrm{e}-0$ \\
\hline \multirow{2}{*}{ Lift coefficient } & $00-100 \mathrm{~s}$ & Growing & 0.621 & $2.6 \mathrm{e}-1$ \\
\hline & $500-600 \mathrm{~s}$ & \pm 0.253 & 0.621 & $3.1 \mathrm{e}-0$ \\
\hline Drag coefficient & $500-600 \mathrm{~s}$ & \pm 0.060 & 1.24 & $1.8 \mathrm{e}-1$ \\
\hline Moment Coefficient & $500-600 \mathrm{~s}$ & \pm 0.196 & 0.621 & $1.9 \mathrm{e}-0$ \\
\hline
\end{tabular}

Table 5: Simulation 1. Results at $6.02 \mathrm{~m} / \mathrm{s}$ flow velocity.

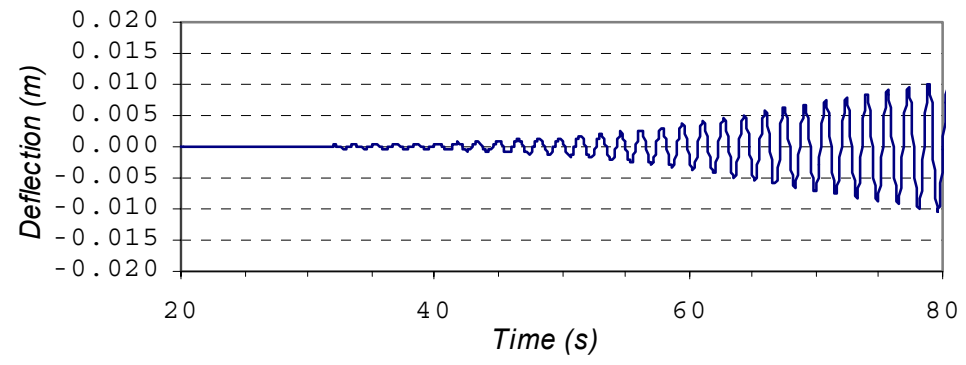

Figure 4: Simulation 1. 20-80s deflection.

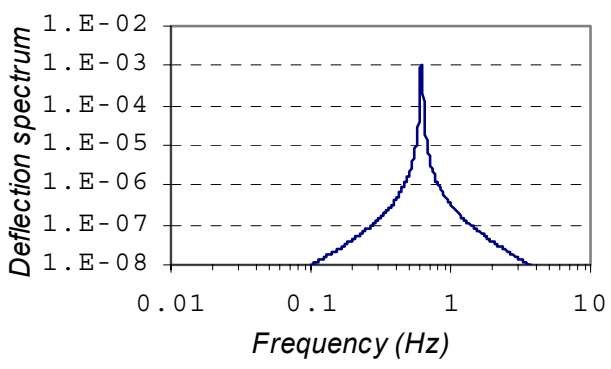

Figure 5: Simulation 1. 20-80s deflection spectrum. 


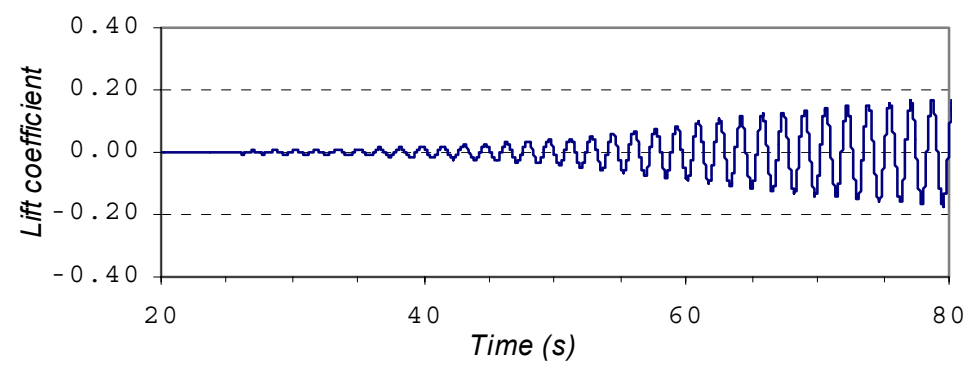

Figure 6: Simulation 1. 20-80s lift coefficient.

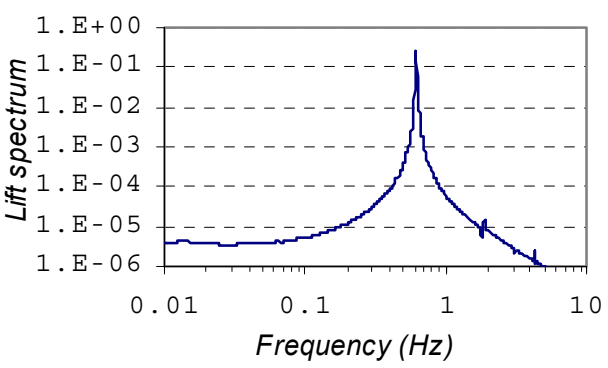

Figure 7: Simulation 1. 20-80s lift spectrum.

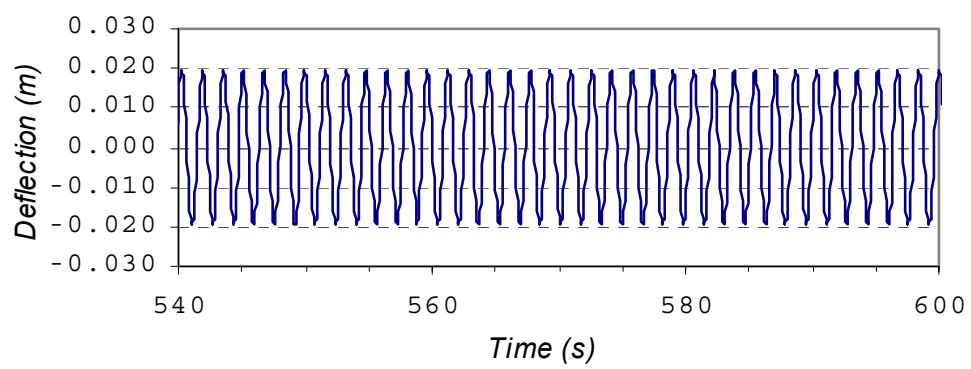

Figure 8: Simulation 1. 540-600s deflection.

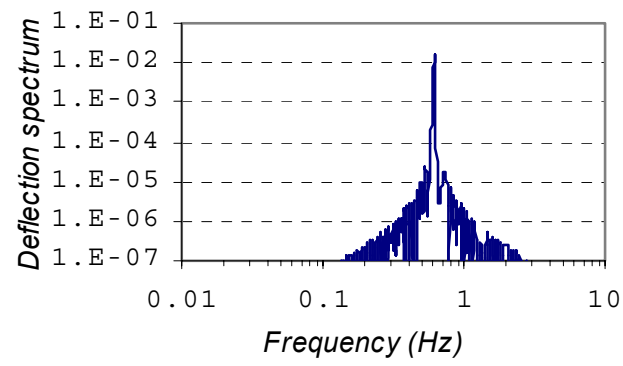

Figure 9: Simulation 1. 540-600s deflection spectrum. 


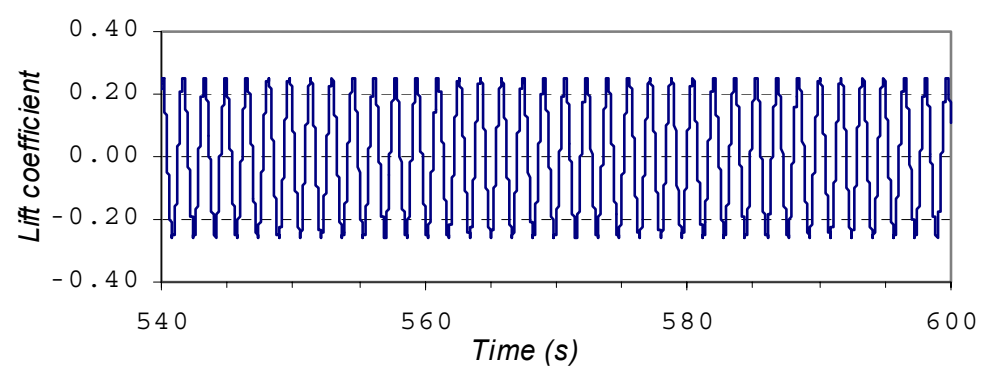

Figure 10: Simulation 1. 540-600s lift coefficient.

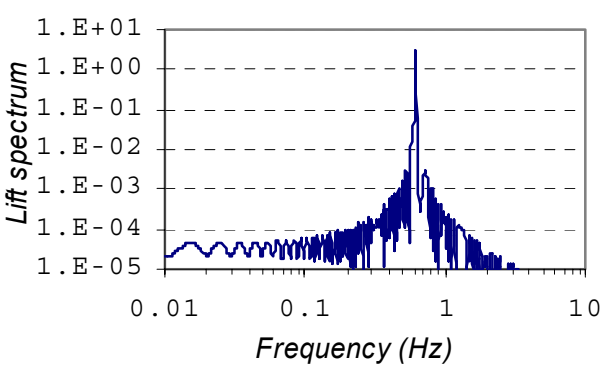

Figure 11: Simulation 1. 540-600s lift spectrum.

Simulation 2: $\mathbf{R e}=\mathbf{4 . 3 7 E 5}$. Now, it will be presented the results concerning the free fluid flow velocity $U=7.03 \mathrm{~m} / \mathrm{s}(\mathrm{Re}=4.37 \mathrm{E} 5)$. As it will be demonstrated later, this velocity corresponds to the maximum oscillation obtained considering the range of synchronisation between aeroelastic forces and the basic structural frequency. The parameters considered in this simulation are equivalent to the first simulation, including the used incremental time steps and the simulation phases. It is presented in table 6 some static values when the flow around a fixed cross-section is considered.

In table 7 and figures 12-19 are presented some of the most significant results concerning displacements and aeroelastic forces at the mid-span section after releasing the structure.

As in the first simulation, after being released, the structure suffers some disturbance, mainly in the horizontal direction, due to drag. In this simulation, this process is prolonged for about 35s. After this phase, the structure remains almost quiet while the lift force acquire enough amplitude to start the vertical oscillation by about 200s. However, this lift force oscillates at correspondent initial Strouhal frequency $(\mathrm{f}=0.692 \mathrm{~Hz})$. After that, the structure begins a consistent increase of the vertical oscillation amplitude until reaching an unalterable value by about 500s time. During this period, the vertical aeroelastic forces develop an adapting process, from the initial Strouhal frequency to the basic structural frequency, elevating also the corresponding amplitude. After 500s time, these both oscillations evidence a constant frequency of $0.62 \mathrm{~Hz}$. The remaining movements and forces oscillate with small amplitude, but they are conditioned by the basic structural frequency. It should be mentioned that the value of the obtained maximum vertical acceleration amplitude is $0.44 \mathrm{~m} / \mathrm{s} 2$. The corresponding derived intensity of perception factor $\mathrm{KB}$ value is 8.9 . 


\begin{tabular}{|c|c|c|c|c|c|c|c||}
\hline$R e$ & $U(m / s)$ & $\Delta t(s)$ & $C F 1$ & $\Delta C F 1$ & $\Delta C F 2$ & $\Delta C F 12$ & $S t$ \\
\hline $4.37 \mathrm{E} 5$ & 7.03 & $2 \mathrm{E}-3$ & 1.122 & 0.0197 & 0.064 & 0.051 & 0.107 \\
\hline
\end{tabular}

Table 6: $\mathrm{Re}=4.37 \mathrm{E} 5$. Relevant values got when the cross-section is at rest.

\begin{tabular}{|c|c|c|c|c|}
\hline Dependent variable & Time interval & Amplitude & $\begin{array}{l}\text { Frequency } \\
(\mathrm{Hz})\end{array}$ & $\begin{array}{l}\text { Max. spec- } \\
\text { trum value }\end{array}$ \\
\hline \multirow{2}{*}{ Deflection } & $200-300 \mathrm{~s}$ & Growing & 0.621 & $1.6 e-5$ \\
\hline & $600-700 \mathrm{~s}$ & $\pm 2.9 \mathrm{e}-2 \mathrm{~m}$ & 0.621 & $3.2 \mathrm{e}-2$ \\
\hline \multirow{2}{*}{ Vertical Acceleration } & $200-300 \mathrm{~s}$ & Growing & 0.621 & $3.6 \mathrm{e}-3$ \\
\hline & $600-700 \mathrm{~s}$ & $\pm 0.44 \mathrm{~m} / \mathrm{s}^{2}$ & 0.621 & $7.5 \mathrm{e}-0$ \\
\hline \multirow{3}{*}{ Lift coefficient } & $00-100 \mathrm{~s}$ & Growing & 0.692 & $2.0 \mathrm{e}-3$ \\
\hline & $300-400 \mathrm{~s}$ & Growing & 0.703 & $1.1 \mathrm{e}-1$ \\
\hline & $600-700 \mathrm{~s}$ & \pm 0.202 & 0.621 & $1.4 \mathrm{e}-0$ \\
\hline Drag coefficient & $600-700 \mathrm{~s}$ & \pm 0.053 & 1.24 & $5.7 e-4$ \\
\hline Moment Coefficient & $600-700 \mathrm{~s}$ & \pm 0.244 & 0.621 & $2.1 \mathrm{e}-0$ \\
\hline
\end{tabular}

Table 7: Simulation 2. Results at $7.03 \mathrm{~m} / \mathrm{s}$ flow velocity.

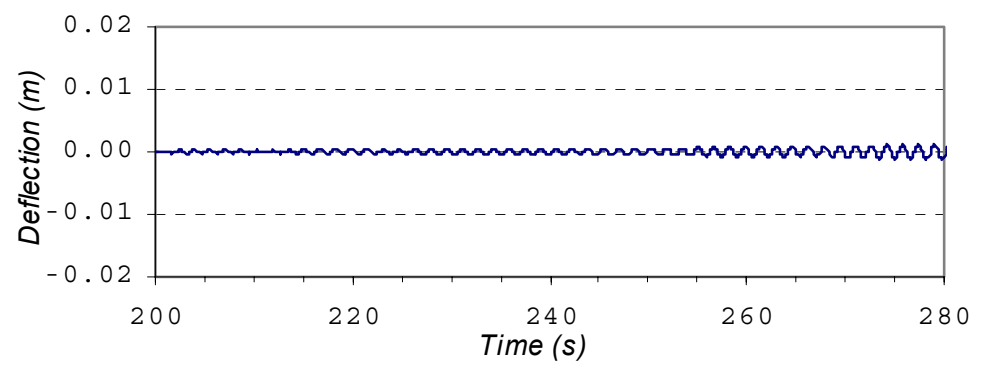

Figure 12: Simulation 2. 200-280s deflection.

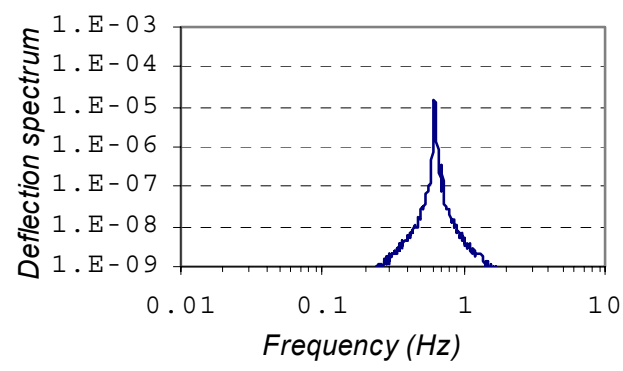

Figure 13: Simulation 2. 200-300s deflection spectrum. 


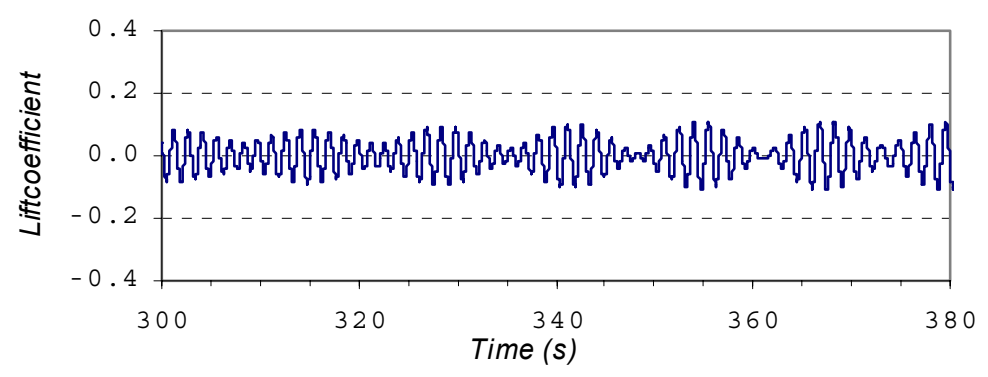

Figure 14: Simulation 2. 300-380s lift coefficient.

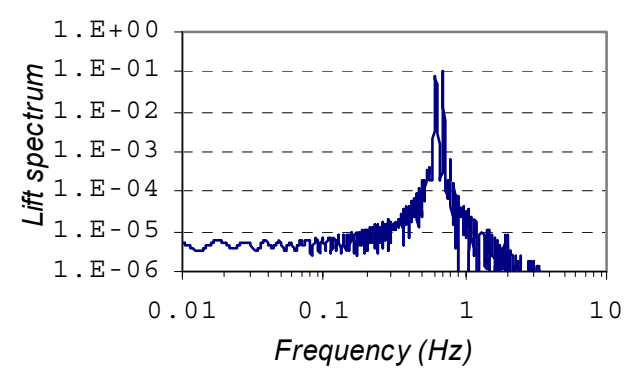

Figure 15: Simulation 2. 300-400s lift spectrum.

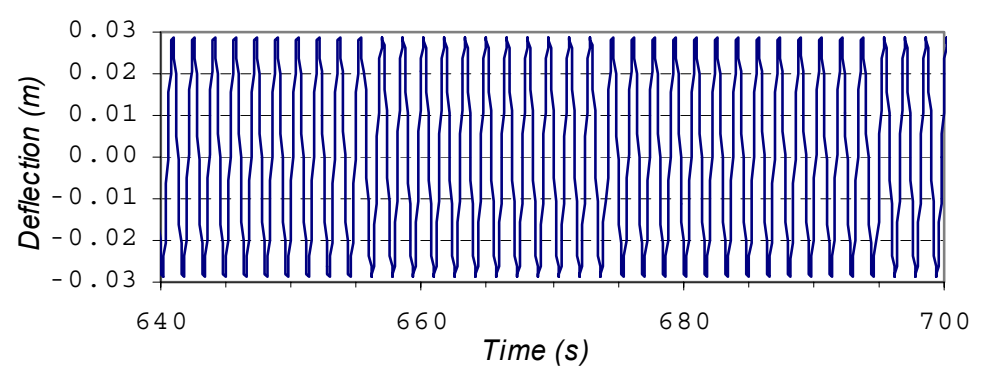

Figure 16: Simulation 2. 640-700s deflection.

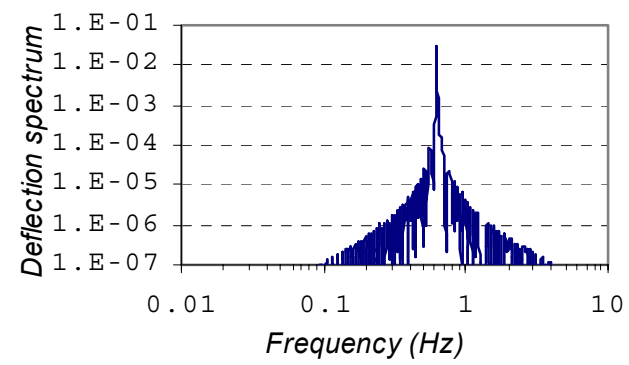

Figure 17: Simulation 2. 600-700s deflection spectrum. 


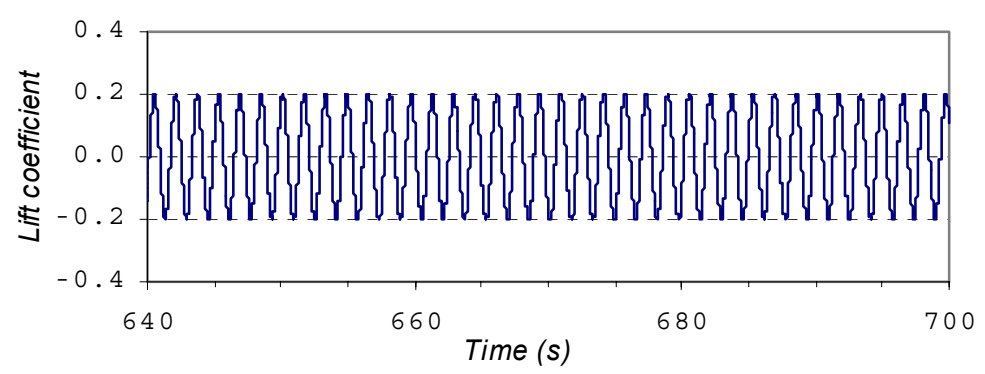

Figure 18: Simulation 2. 640-700s lift coefficient.

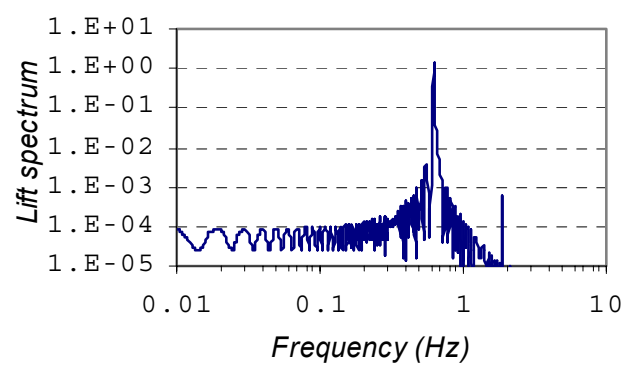

Figure 19: Simulation 2. 600-700s lift spectrum.

Based on the above results, it is possible to draw the following preliminary conclusions:

- For the considered velocities of the free fluid flow, no mechanism of instability (growing amplitudes) was observed;

- Considering these two simulations, it is observed a small perturbation due to the aeroelastic drag forces after liberating the presented structure, which takes less than one minute of simulation;

- For the presented structure under considered wind actions, the oscillations' stability was quickly reached, which takes less than 9 minutes of simulation;

- The desired synchronisation was reached for different free flow Strouhal velocity. Considering this velocity, the time step needed to reach the synchronisation is longer, but the oscillation amplitude is also higher.

It will be important to note that the horizontal perturbation of the structure is consequence of the adopted methodology, and it is not a real fact. However, this perturbation does not obstruct the important issue of this work, which deals with the evaluation of the accelerations of movements for the analysis of serviceability conditions.

\subsection{Analysing the range of synchronisation}

Considering the basic frequency of the given structure, one tries to find out the range of free fluid velocities where the lock-in phenomena happens, i.e. where the synchronisation between aeroelastic forces and movements turns out. The important issue consists in identifying the velocity range in order to find out the maximum amplitude of oscillations. Alternatively, one can evaluate the oscillation amplitude for different velocities, close to the Strouhal velocity, so that the mentioned maximum amplitude of oscillations can be located. The results 
evaluated for the RMS value of the acceleration amplitude, at mid-span section, considering several free flow velocities are illustrated in the figure 20. At $\operatorname{Re}=4.37 \mathrm{E} 5(\mathrm{U}=7.03 \mathrm{~m} / \mathrm{s})$ was taken the RMS maximum amplitude $0.31 \mathrm{~m} / \mathrm{s}$, which corresponds to a peak acceleration of $0.44 \mathrm{~m} / \mathrm{s}$. One can also see that the range where it was possible to get the synchronisation extents from $\mathrm{Re}=3.8 \mathrm{E} 5$ to $\mathrm{Re}=4.6 \mathrm{E} 5$. It is worth to remember that, in this case the Strouhal velocity is $\mathrm{Re}=4 \mathrm{E} 5$. Furthermore, the maximum acceleration amplitude is obtained relatively far from the Strouhal velocity and, after that, this amplitude almost vanishes.

Figure 21 shows another important characteristic of this example: the evolution of the evaluated RMS lift coefficient CF2. In this figure, one can see that the maximum amplitude of aeroelastic force was taken from the Strouhal frequency. Moreover, there is a small range where this amplitude decreases while the amplitude of movements increases.

Additionally, it will be important to know how difficult it is to get the mentioned synchronisation, i.e.; When does it start? When is it complete? And how long does it take? It is important to remember that initially the structure was fixed until the free flow velocity reaches the desired value, and after that it is liberated. This procedure causes a small horizontal perturbation that can not be forgotten. So, it is shown in the figure 22 the synchronisation starting time, the synchronisation initial time and the corresponding time step. By starting time it is understood the instant when the amplitude reaches $5 \%$ of the maximum amplitude. In the same way, the initial time will be the instant when the amplitude reaches $95 \%$ (well developed) of maximum amplitude. The time step is the period between the last two times. One can see that the starting time, the initial time and the time step increase. At the maximum movements' amplitude, the time needed to have a well developed synchronization is almost 9 minutes in this case.

To finish the presentation of results, it will be important to refer that the aerodynamic coefficients, evaluated considering the fixed obstacle, slowly decrease in the considered range. For example, the RMS lift coefficient CF2 decreases from 0.055 to 0.038 .

Based on the above results, it is possible to write down the following conclusions:

- The interval of fluid flow velocity where the synchronisation between aeroelastic forces and movements turns out is quite large;

- Along the considered range, the movement's amplitude grows almost until the end, but the matching aeroelastic forces decrease from the set up velocity according to the basic structural frequency and Strouhal frequency $(U=6.02 \mathrm{~m} / \mathrm{s})$;

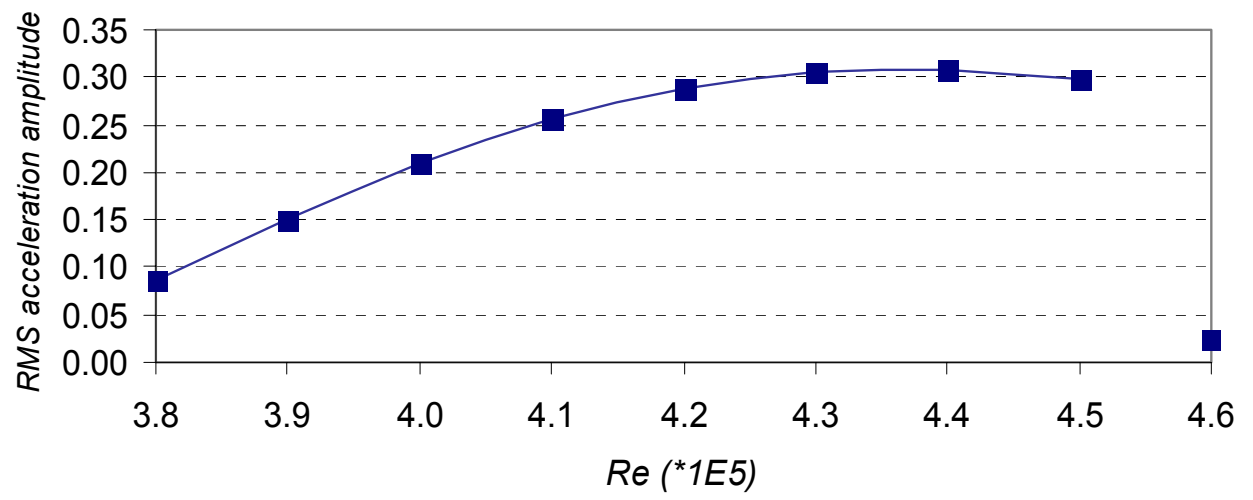

Figure 20: RMS acceleration amplitude in the synchronisation range of basic frequency. 


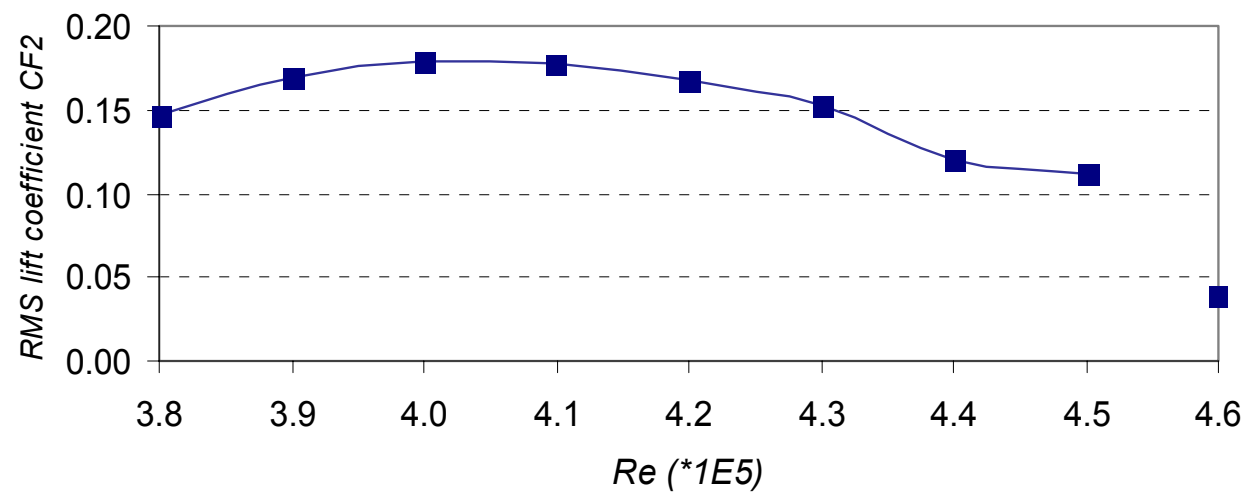

Figure 21: Evolution of the RMS lift coefficient in the synchronisation range of basic frequency.

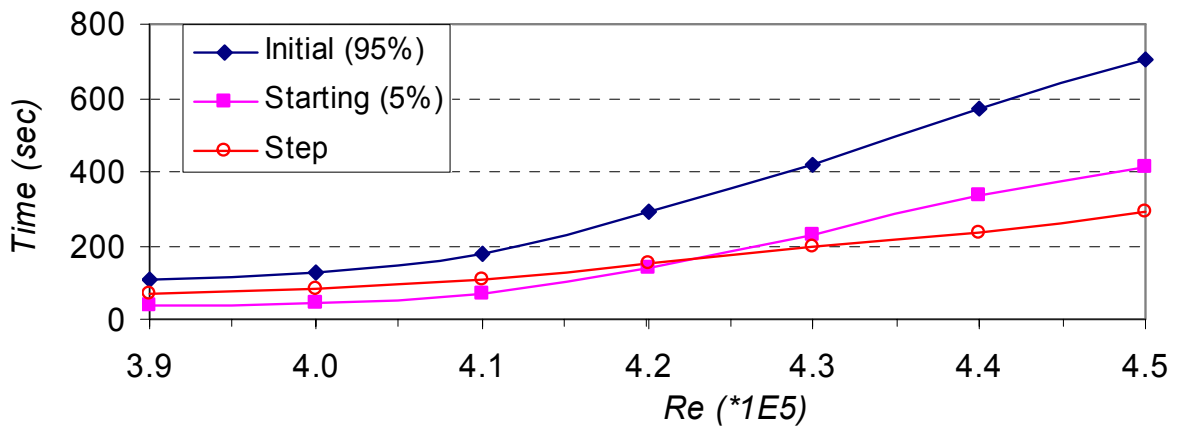

Figure 22: Step time evaluated in the synchronisation range of basic frequency.

- The maximum peak accelerations' amplitude $\left(0.44 \mathrm{~m} / \mathrm{s}^{2}\right)$ was evaluated at a velocity $(U=7.03 \mathrm{~m} / \mathrm{s})$ relatively far from the set up Strouhal velocity and is $50 \%$ higher than that one;

- During the considered range of the free fluid flow, the time needed to get the synchronisation has an important increase;

Taking into consideration the above simulations, it is possible to compare the evaluated results with limit-values indicated in the available literature from human comfort point of view $[9,10,11,12,13]$, as it is indicated in table 1 . With this in mind, and remembering that there are not any definite limits of acceptability for a specific situation, as the analyzed one, it is possible to conclude the following:

- The evaluated maximum amplitude of accelerations, calculated for the first example (1st vertical frequency), is $0.44 \mathrm{~m} / \mathrm{s} 2$ that corresponds to $4.4 \% \mathrm{~g}$, or $3.1 \% \mathrm{~g}$ in terms of RMS, which can be classified as an annoying case or a perceptible case;

- If one were to consider the corresponding derived intensity of perception factor KB values, proposed by DIN 4150, this simply supported bridge does not verify the specified limit. It should be noted that the value of the first frequency is out of the defined range as well as in the ISO 2631 proposal;

- For this specific structure and for the range tested cases, it was possible to synchronise all the aeroelastic forces with the corresponding structural frequencies. 


\section{CONCLUSIONS}

The results presented here illustrate an application of a new numerical methodology for the analysis of serviceability condition of bridge structures under wind action. Generally, this kind of aeroelastic phenomenon does not lead to any mechanism of instability, but can generate human body discomfort when the amplitude of acceleration reaches certain limit values. Typically, the amplitude of acceleration is high if both forces and displacements have a particular frequency of oscillation, i.e. when the phenomenon lock-in takes place.

In this case, it is quite large the interval of fluid flow velocity where the synchronisation between aeroelastic forces and movements turns out according to the basic frequency. The maximum peak accelerations' amplitude $(0.44 \mathrm{~m} / \mathrm{s} 2)$ was evaluated at a velocity $(U=7.03 \mathrm{~m} / \mathrm{s})$ relatively far from the set up velocity according to the basic structural frequency and to the Strouhal frequency $(\mathrm{U}=6.02 \mathrm{~m} / \mathrm{s})$. That value is $50 \%$ higher than this one. Moreover, at the same time the matching aeroelastic forces decrease and the time needed to get the synchronisation has an important increase.

Considering the wind action, the analysed simply supported structure, with a rectangular deck cross section $(B / D=6)$, which is not so sensitive to flutter [6], can lead to some human discomfort, once that the evaluated maximum amplitude of accelerations is $0.44 \mathrm{~m} / \mathrm{s} 2$. This fact indicates an unsatisfactory behaviour from the point of view of serviceability limit states of vibrations.

Although the above conclusions drawn from the specific case of a simply supported bridge, with a rectangular deck cross section, can not be directly extrapolated to other situations, the methodology presented in this paper can be applied to other cases with different shapes of the deck cross section.

It is still worth mentioning that it will be important to have specific rules in terms of characteristics of incoming fluid flow, serviceability limit states definition and maximum time period of analysis, in order to evaluate this kind of lock-in phenomenon in bridges field.

\section{REFERENCES}

[1] E. Simiu, R. Scanlan, Wind effects on structures. An introduction to wind engineering, John Wiley \& Sons, 1986.

[2] R. H. Scanlan, J. J. Tomko, Airfoil and Bridge deck flutter derivatives, Journal of the Engineering Mechanics Division, ASCE, vol. 97 (6), 1971.p. 1717-1737.

[3] N. P Jones, R. H. Scanlan, Advances (and challenges) in the prediction of long-span response to wind, Proceedings of the international symposium on advances in bridge aerodynamics, Copenhagen, Denmark, 1998. p. 59-85.

[4] A. V. Lopes, Aplicação da Dinâmica Computacional de Fluidos à Análise Aeroelástica de Estruturas Esbeltas, Ph.D. Thesis, Dep. Eng. Civil, FCTUC, Coimbra, Portugal, 2001.

[5] A. V. Lopes, A. Cunha, L.M.C. Simões, Aeroelastic analysis of a slender bridge deck based on a CFD algorithm, Proceedings of the 4th International Conference on Structural Dynamics - EURODYN2002, Munich, Germany, 2002. p. 575-580.

[6] A. V. Lopes, A. Cunha, L. M. C. Simões, A Nonlinear Coupled Fluid-Structure Aeroelastic Analysis of a Slender Bridge Deck, International Journal for Numerical Methods in Fluids, 45(5), 2004. p. 527-553. 
[7] Eurocode 1, Basis of design and actions on structures, part 2.4, Wind Loads, 1991.

[8] A. Larsen, S. Esdahl, J.E. Andersen, T. Vejrum, H.H. Jacobsen, Vortex shedding excitation of the Great Belt suspension bridge, $10^{\text {th }}$ Int. Conf. Wind Engineering into the $21^{\text {st }}$ Century, Denmark, 1999.

[9] H. Bachmann, A. Walter, Vibrations in structures induced by man and machines, Zurich, IABSE, 1987.

[10] International Standards ISO 2631, Mechanical vibration and shock Evaluation of human exposure to whole-body vibration, vol.1, General requirements, 1989.

[11] Bulletin D'Information $\mathrm{N}^{0}$ 209, Vibration Problems in Structures, Comité EuroInternational du Béton CEB, 1991.

[12] German Institute for Standards, DIN 4150, Vibrations in Civil Engineering - Part 2: Effects on people in buildings, Berlin, 1975.

[13] National Research Council of Canada, Supplement to the National Building Code of Canada, Ottawa, 1990.

[14] S. V. Patankar, Numerical Heat Transfer and Fluid Flow, Hemisphere Publishing Corporation, 1980.

[15] H. K. Versteeg, W. Malalasekera, An Introduction to Computational Fluid Dynamics, Longman Malaysia, 1995.

[16] J. H. Ferziger, M. Peric, Computational Methods for Fluid Dynamics, Springer-Verlag, Berlin Heidelberg, 1996.

[17] W. Rodi, Turbulence Models and Their Application in Hydraulics - A State of the Art Review, International Association for Hydraulic Research, Delft, Netherlands, 1980.

[18] H. Tennekes, J. L. Lumley, A first Course in Turbulence, Sixth Printing, Massachusetts Institute of Technology Press, Massachusetts, 1980.

[19] M. S. Hossain, W. Rodi, A turbulence Model for Buoyant Flows and its application to Vertical Buoyant Jets, Turbulent Buoyant Jets and Plumes, Pergamon Press, Oxford, 1982. p. 121-178.

[20] L. A. Oliveira, Modelação Numérica de Escoamentos em Regime Turbulento, Dep. Eng. Mecânica, FCTUC, Coimbra, 1990.

[21] Klaus-Jürgen Bathe, Finite Element Procedures in Engineering Analysis, Prentice-Hall, New Jersey, 1982.

[22] R. W. Clough, J. Penzien, Dynamics of Structures, McGraw-Hill, 1993.

[23] O. C. Zienkiewicz, R. L. Taylor, The Finite Element Method, McGraw-Hill, 1989.

[24] E. Naudascher, D. Rockwell, Flow-Induced Vibrations, An engineering guide, A. A. Balkema, Rotterdam, Netherlands, 1994.

[25] International Standards ISO 4866, Mechanical vibration and shock - Vibration of buildings - Guidelines for the measurement of vibrations and evaluation of their effects on buildings, 1990.

[26] A. V. Lopes, A. Cunha, L.M.C. Simões, Numerical evaluation of shape coefficients and flutter derivatives based on a CFD algorithm, Fifth World Congress on Computational Mechanics, Vienna, Austria, 2002. p. I-605. 